\title{
Is that a polarimeter in your pocket? A zero-cost, technology- enabled demonstration of optical rotation
}

Patrick I. T. Thomson*

University of Strathclyde, Department of Pure \& Applied Chemistry, 295 Cathedral Street, Glasgow,

$5 \quad$ G1 $1 X L$

\begin{abstract}
A polarimeter can be constructed using a document camera as a visualizer and a standard

smartphone screen as a source of polarized light. A pair of 3D glasses can be used as a polarizing filter and the optical rotation angle of liquids can be measured using any free compass app. Consequently, a demonstration of optical rotation can be conducted with no preparation time or specialized equipment.
\end{abstract}

\section{GRAPHICAL ABSTRACT}

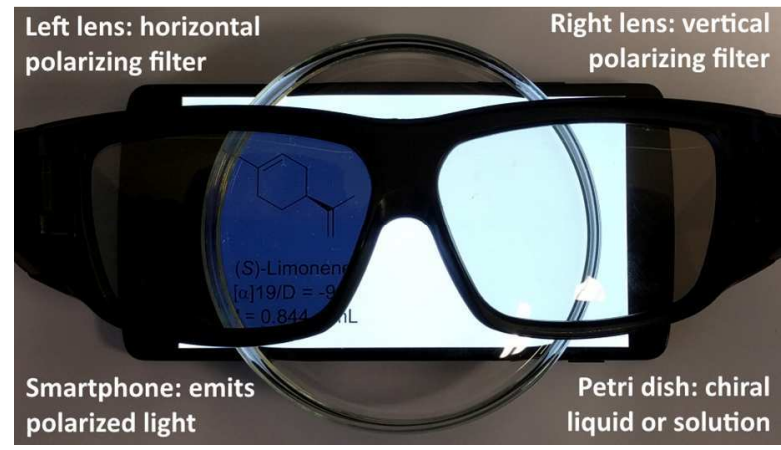

\section{KEYWORDS}

Audience: First-Year Undergraduate

Domain: Demonstration, Organic Chemistry

Pedagogy: Hands-on Learning, Manipulatives

Topic: Chirality, Optical Activity

Practical demonstrations, conducted by a lecturer in front of an audience, are as old as science 20 teaching itself. ${ }^{1}$ Demonstrations involving the transmission of light, for example solution-state color changes in chemistry, have been conveniently presented to large audiences using overhead projection technology for many decades. ${ }^{2,3}$ One area that this has been applied to with great success is that of optical rotation: The phenomenon of the rotation of plane polarized light by molecules that exhibit 
chirality. ${ }^{3-15}$ Such demonstrations can be readily converted into hands-on experiments, ${ }^{16-21}$ but the

requirement for specialized or self-constructed equipment presents a barrier to entry.

The measurement of the plane of polarized light requires the use of an instrument called a polarimeter. A polarimeter consists of a source of monochromatic light, a polarizing filter, an area where this light can pass through a sample, a second rotatable polarizing filter (the analyzer), and a detector to measure the intensity of the resulting light and consequently the angle of rotation. In the absence of a sample, the light transmission will be maximized when the polarizers are aligned, and minimized when the polarizers are perpendicular to each other. Chiral molecules rotate the plane of polarized light by an angle dependent on temperature, concentration, path length, wavelength of light, and the specific rotation of the sample. This angle can be measured by rotating the analyzer to minimize or maximize the transmitted light, then converted to a specific rotation.

Literature values for specific rotation are cited at a particular temperature and monochromatic wavelength, most commonly $589 \mathrm{~nm}$. Specific rotation is different at different wavelengths, so for this reason, measurements taken in the lecture theatre using white light sources also exhibit optical rotatory dispersion (colored fringes of light on either side of the angle of minimum transmission). ${ }^{5}$ Demonstrations, then, are usually limited to qualitative observations, such as the equal and opposite optical rotation of enantiomers, or the modification of chiral activity during a solution-phase reaction..$^{7,22}$

Demonstrations also require the use of polarizing film, and equipment to hold and rotate this film. For these demonstrations, polarizing film has been sourced from certain types of consumer electronics, ${ }^{16}$ certain kinds of sunglasses, ${ }^{23-25}$ or purchased from scientific laboratory suppliers. The holder can be constructed from inexpensive materials, ${ }^{16}$ but combined time and effort remains a barrier to entry for a simple lecture demonstration. Additionally, the traditional overhead projector typically contains a high-powered incandescent light source which can also pose a fire hazard when using flammable solvents.

The current demonstration is one which can be assembled extremely quickly from entirely 50 household items, namely a smartphone and a pair of 3D glasses. It can be displayed using the nowubiquitous lecture support, the document camera. And it can be used to measure household 
1

2

3

4

5

6

7

substances: solutions of sugar. Demonstrations of chemical principles created from mundane items are more engaging for students, and reduce the perceived abstract nature of the principle being demonstrated. ${ }^{26}$ A diagram of the assembly of the polarimeter is shown below (Figure 1), where the 55 smartphone acts as both source of light and a rotatable polarizing filter. The 3D glasses act as the second polarizing filter, and the document camera acts as a method of measuring and displaying the angle of rotation. A video of the demonstration being used in a lecture is available in the Supporting Information.

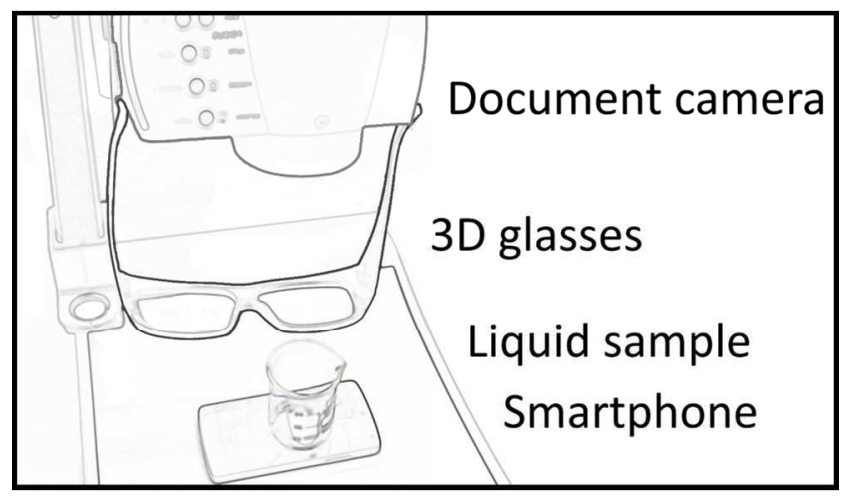

Figure 1: Schematic of the polarimeter

\section{COMPONENTS OF THE POLARIMETER}

To run this demonstration, the following materials and equipment are required: A source of polarized light (usually a smartphone), a pair of 3D glasses, a document camera, a chiral liquid or solution of a chiral molecule (usually sucrose), and a way of measuring angles (a protractor, or a compass application installed on the smartphone).

\section{Light source: the smartphone}

The first item of technology used in this demonstration is the smartphone. Smartphones are a mainstay of the student pocket and higher education practitioners and researchers have been quick to capitalize on this, with such advances as in-lecture polling, student video production, and lab notebooks. ${ }^{27-30}$ However, as well as a platform for digital and visual content, the smartphone has physical hardware capable of specific interactions with the chemical world. For example, the camera has been used as a colorimeter for spectroscopy, and the audio output has been used to control an electroformation chamber. ${ }^{31,32}$ Of relevance to the current research, mobile phone screens are polarized light sources. ${ }^{24}$ A liquid crystal display (LCD) emits polarized light as a necessary by-product 
of its technology, ${ }^{33,34}$ but polarizing film is a component of more modern organic light-emitting diode (OLED) displays as well in order to eliminate internal reflection and glare. ${ }^{35}$ It seems, therefore, that pocket polarized light sources are everywhere, and here to stay, although some newer models of phone screens use circular polarizing filters which need a modification to the current demonstration (see Supporting Information). Particular acknowledgement at this point is given to the recent prior work of Schwartz et. al. ${ }^{24}$ who were the first to use the same LCD as a source of light and a polarizing filter simultaneously. A tablet computer or laptop screen can also be used if no suitable smartphone is available.

\section{Polarizing filter: the 3D glasses}

The second item of technology used in this demonstration is a pair of stereoscopic glasses for use in 3D cinema. There are two widespread types of 3D cinema technology at the time of writing, corresponding to two types of polarization: linear polarization (used in $3 \mathrm{D}$ IMAX ${ }^{\circledR}$ screens) and circular polarization (used in other types of 3D cinema). IMAX ${ }^{\circledR}$-compatible $3 \mathrm{D}$ glasses can consequently be used as linear polarizers without modification, and can be purchased cheaply or obtained after watching a film. ${ }^{36}$ The circular polarizers found in "RealD-3D" glasses can be converted to linear polarizing film by peeling off the inside layer with a razor blade, or potentially used as-is as part of a demonstration of circular dichroism. If you have 3D glasses of unknown technology, they can be worn while examining a suitable smartphone: The screen will fade to $100 \%$ black on rotation only when viewed through linear polarizers. Certain kinds of anti-glare sunglasses have also been used in prior demonstrations. ${ }^{23-25}$

95 Analyzer: the document camera

The third item of technology used in this demonstration is the visualizer, or document camera. This relatively new article of technology has fast become an essential part of the education repertoire when paired with a digital projector. In chemical education, it has been used to demonstrate chemical reactions, ${ }^{37}$ color changes, and even optical rotation. ${ }^{24}$ Historically, an overhead projector (OHP) was used for the same purpose,$^{2}$ and optical rotation was one of the few demonstrations that was easier with the older technology, owing to the OHP's built-in light source. ${ }^{38}$ However, the document camera is a more versatile tool, as it can record and project non-transparent demonstrations onto screens of 
1

2

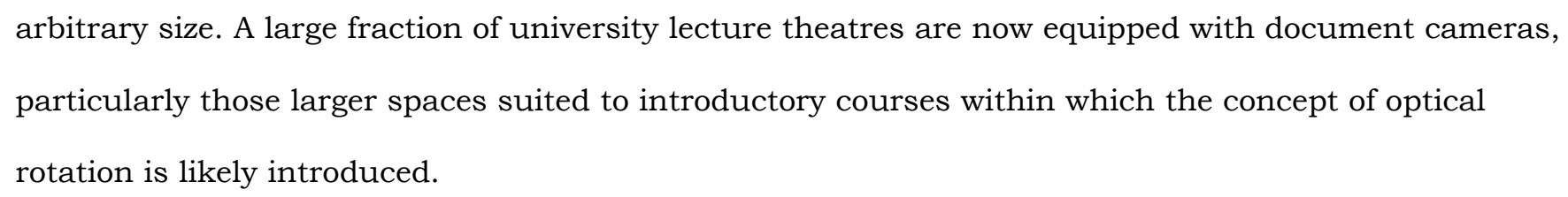

arbitrary size. A large fraction of university lecture theatres are now equipped with document cameras, particularly those larger spaces suited to introductory courses within which the concept of optical rotation is likely introduced.

\section{RUNNING THE DEMONSTRATION}

3D glasses containing linearly-polarizing filters are affixed to a document camera using adhesive tape, in such a way as to cover most of the field of view of the camera with one lens of the glasses. Transparent contact adhesive tape leaves no residue, which may avoid causing undue distress to the learning technology team of your institution (the glasses could also be clamped using a retort stand).

A smartphone is placed on the bed of the visualiser and brought into focus (at this point, automatic focusing should be disabled if possible). The screen is set to display an all-white (or pure colored) background using a flashlight app, blank word processor, or other source of single color. A sample of chiral liquid is placed in a beaker or measuring cylinder and placed on the screen of the smartphone. A solution of table sugar (sucrose) is the recommended substrate for this demonstration, primarily due to safety concerns but also since aqueous solutions can be prepared during the lecture to further enhance the demonstration. ${ }^{26,39}$

When used as part of a more comprehensive lesson on chirality, limonene, pinene, and carvone all exist as pairs of enantiomers which can be discriminated by scent and so the same materials can be used for visual and olfactory demonstrations of chirality. If both enantiomers of a chiral liquid are available, then they can be mixed during the demonstration to show the reduction of optical activity in non-enantiopure or racemic mixtures. Care should be taken when using these substances, however, as they are flammable and may have other specific chemical hazards (see Hazards section for further details).

The smartphone can then be rotated back and forth between the angle where the unaltered light is minimized (Figure 2, left), and one when the light passing through the sample is minimized (Figure 2, right). A particularly impactful and quick demonstration could be to load an image of a chemical structure, then twist the smartphone so that the image is completely occluded by the action of the perpendicular polarizing filter. A beaker can then be placed on top of the phone and a chiral liquid 
poured in, bringing the image back into view. The phone can then be rotated back and forth to demonstrate the change in angle of maximum occlusion.
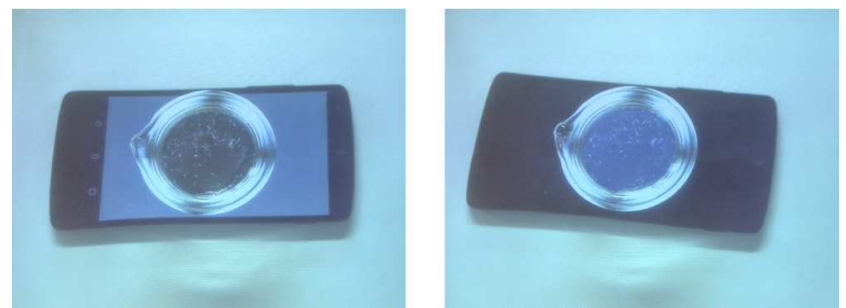

Figure 2: A dilute solution of sucrose exhibiting a rotation of ca. $5^{\circ}$. The phone was rotated to minimize light from the screen (right) and the sample (left). A video of this in action is available in the Supporting Information.

To measure angles, the demonstration can be carried out on a sheet of plain paper and the edge of the smartphone used as a ruler to draw straight lines. The angle can then be measured using any free compass application running on the same phone (usually accurate to within a few degrees). ${ }^{40}$ If the smartphone is not equipped with a magnetic field sensor, then a protractor can be used to measure the angle of rotation.

Using this setup, the measured rotation of a sample of $(R)$-limonene of depth $0.4 \mathrm{dm}$ was found to be $+56^{\circ}$, which corresponds to a specific rotation of $+166^{\circ} .(R)$-limonene was chosen as it is the more common isomer, and cheaply available from commercial sources (see Supporting Information for more sample data and details of calculations). If this demonstration is to be conducted by students directly, a sucrose solutions should be used instead as this poses no fire hazard.

The measured specific rotation of $(R)$-limonene is much higher than the literature value of $+120^{\circ}$ at $589 \mathrm{~nm}$, due to the aforementioned optical rotatory dispersion as the white light of the smartphone has a significant blue component, as shown in Figure 3 below.

\section{HAZARDS}

Handling: Limonene, pinene, and carvone are irritants and students should not be allowed to work with these solvents. The relevant material safety data sheet should be consulted before use and institutional risk-assessment procedures should be followed prior to any demonstration. Limonene, pinene, and carvone are also flammable, so care should be taken in line with institutional procedures as with any flammable solvent. These solvents should only be handled by an experienced chemist 
1

wearing a lab coat, safety glasses, and gloves. Sucrose solutions should be used instead whenever possible, to demonstrate qualitative effects.

Disposal: Sugar solutions can be disposed of down the sink. Organic solvents limonene, pinene and carvone should be disposed of in line with your institutional practices for non-halogenated solvents, or kept for re-use at a later date.

Further handling advice can be found in the Supporting Information.

\section{OPTICAL ROTATORY DISPERSION}

Optical rotatory dispersion is the phenomenon whereby the optical rotation of a sample depends on the wavelength of the polarized light. ${ }^{41,42}$ Since classroom light sources are generally polychromatic, demonstrations using classroom light sources have only been used to display the qualitative effects of optical rotation to date. However, numerous prior authors have noted that you can observe optical rotatory dispersion in the form of blue and red tinges to the transmitted light of a sample. $10,17,22,25,43,44$ These demonstrations usually used a broad-spectrum light source, such as the filament bulb of an overhead projector, although some have used colored filters to narrow the spectrum of the light used. ${ }^{45}$

The modern smartphone screen consists of red, green, and blue pixels. To emit white light of the type used in our demonstration so far, all three colors of these pixels are illuminated. Due to variation in the underlying technology, the spectral responses of these pixels are not consistent across different models of smartphone, so the optical emission spectra of the author's own phone was measured whilst displaying red, green, blue, and white images (Figure 3). The spectral trace corresponding to white light approximates a direct superposition of the other three, indicating that individual color families of pixels are being activated by the images (although a small amount of blue light is present in each image). 
Figure 3: The visible light emission of a Nexus 5 smartphone screen, displaying various colors of images, taken with a Si-Photonics CCD array UV-Vis spectrophotometer. Experimental details can be found in the Supporting Information.

The specific rotation of a sample of $(R)$-limonene was then measured using the protocol in the previous section, using red, green, blue, and white light, and charted, along with literature values at various wavelengths and a value taken of the same sample using a manual Hilger-Watts polarimeter (Figure 4). ${ }^{46}$ It can see from the presented trend that even though the colored light sources have a full width at half-maximum (FWHM) of $40-100 \mathrm{~nm}$, the rotational dependence on wavelength is visible the rotation angle of limonene is almost doubled when switching from red to blue light. White light gives a specific rotation of $166^{\circ}$, which corresponds to an effective monochromatic wavelength of $\sim 500$ $\mathrm{nm}$ or a black-body radiation temperature of 5500K, about the same as that for a normal LCD screen.

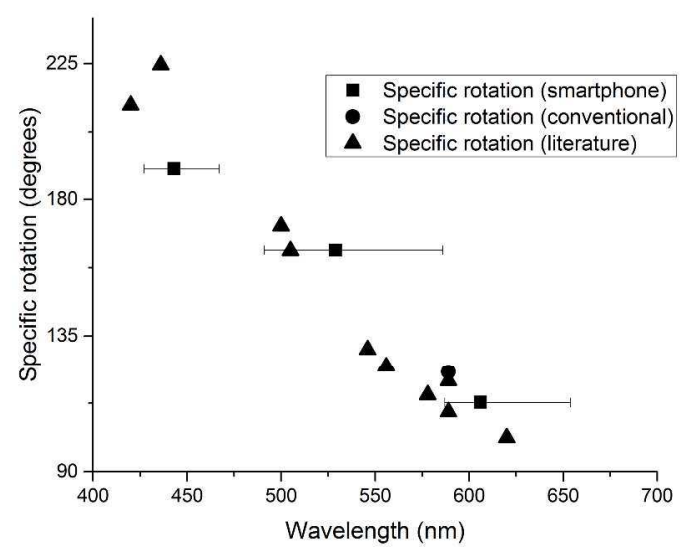

Page 8 of 11 
Figure 4: Wavelength dependence of the specific rotation of $(R)$-limonene, measured with a Nexus 5 smartphone. Path length was $4 \mathrm{~cm}$, and specific rotation was calculated using the literature density of $0.842 \mathrm{~g} / \mathrm{mL}$. Horizontal bars indicate the full width half maximum of the spectral responses taken from Figure 3.

There is scope for further work around wavelength-specific optical rotation - for example, can an accurate monochromatic optical rotation value be calculated, given only a broad-spectrum light source and a single measurement taken with this light?

\title{
CONCLUSION
}

This demonstration has been used with first-year introductory organic chemistry lectures of several hundred students, and could easily be adapted to a hands-on laboratory experiment where students use their own smartphones to take optical rotation measurements. My hope is that this demonstration will be useful in the teaching of optical rotation without time or expense, and will further promote the use of practical demonstrations in large-group teaching.

\author{
ASSOCIATED CONTENT \\ Supporting Information \\ Additional photographs of experimental setup. Video of demonstration. Assessment of chiral \\ liquids and various computer and mobile device screens, additional safety and handling information, \\ details of emission spectra measurements.
}

\section{AUTHOR INFORMATION \\ Corresponding Author \\ *E-mail: patrick.thomson@strath.ac.uk}

\section{ACKNOWLEDGMENTS}

Thanks should be given to Chris Dodds for the use of a UV spectrophotometer and Brian McMillan for the use of a polarimeter, and both for many helpful comments and suggestions throughout the writing process. The author would also like to acknowledge the operational staff development unit at the University of Strathclyde, OSDU, for running a paper-writing challenge that provided the initial encouragement for the current work, and to Peter Kirsop for encouragement during the gestation of the initial idea. Finally, a sincere thanks to the editor and to the reviewers for general and specific feedback during the review process. 


\section{REFERENCES}

1. Thomas, J. M., Michael Faraday and The Royal Institution: The Genius of Man and Place (PBK). CRC Press: 1991.

2. Kolb, D., Introduction to overhead projector demonstrations. J. Chem. Educ. 1987, 64 (4), 348.

3. Dorn, H. C.; Bell, H.; Birkett, T., A simple polarimeter and experiments utilizing an overhead projector. $J$. Chem. Educ. 1984, 61 (12), 1106.

4. Knauer, B., A demonstration of the optical activity of a pair of enantiomers. J. Chem. Educ. 1989, 66 (12), 1033.

Hambly, G. F., Optical activity: an improved demonstration. J. Chem. Educ. 1988, 65 (7), 623.

6. Meloan, C. E., A Demonstration to let students see optical rotation. J. Chem. Educ. 1978,55 (5), 319. Hill, J. W., An overhead projection demonstration of optical activity. J. Chem. Educ. 1973, 50 (8), 574. Evans, J. O. M.; Tietze, H. R., Optical rotation. J. Chem. Educ. 1964, 41 (12), A973.

9. Spear, C. S., A demonstration polarimeter. J. Chem. Educ. 1960, 37 (4), 203.

10. Noller, C. R., Apparatus for lecture demonstration of optical activity. J. Chem. Educ. 1949, 26 (5), 269.

11. Burkett, H., A demonstration polarimeter. J. Chem. Educ. 1949, 26 (5), 273.

Garvin, J. E., Inexpensive polarimeter for demonstrations and student use. J. Chem. Educ. 1960, 37 (10), 515.

13. Frank, F. J.; Kidwell, S. M., Polarimeter for an overhead projector. J. Chem. Educ. 1969,46 (1), 58.

14. Kapauan, A. F., A simple split-field polarimeter. J. Chem. Educ. 1973, 50 (5), 376.

15. Breton, G. W., An Inexpensive Homemade Polarimeter for Demonstration Use in the Classroom. Journal of Laboratory Chemical Education 2015, 3 (1), 7-11.

16. Mehta, A.; Greenbowe, T. J., A Shoebox Polarimeter: An Inexpensive Analytical Tool for Teachers and Students. J. Chem. Educ. 2011, 88 (8), 1194-1197.

17. Vaksman, M. A.; Lane, J. W., Using Guided Inquiry to Study Optical Activity and Optical Rotatory Dispersion in a Cross-Disciplinary Chemistry Lab. J. Chem. Educ. 2001, 78 (11), 1507.

18. Nechamkin, H., A student polarimeter. J. Chem. Educ. 1954, 31 (11), 579.

19. Shaw, W. H. R., A mailing-tube polarimeter. J. Chem. Educ. 1955, 32 (1), 10.

20. Vennos, M. S., Construction and uses of an inexpensive polarimeter. J. Chem. Educ. 1969,46 (7), 459.

21. Kiplinger, C. C., An improvised polarimeter. J. Chem. Educ. 1930, 7 (9), 2174.

22. Dean, W. K., Addendum to "Simple demonstration of optical activity". J. Chem. Educ. 1977, 54 (8), 494.

23. Levine, S. G., A classroom demonstration polarimeter. J. Chem. Educ. 1990, 67 (12), 1064.

24. Schwartz, P. M.; Lepore, D. M.; Morneau, B. N.; Barratt, C., Demonstrating Optical Activity Using an iPad. J. Chem. Educ. 2011, 88 (12), 1692-1693.

25. Munchausen, L. L., Polarization of Scattered Light. J. Chem. Educ. 1994, 71 (2), 155.

26. Monteiro, N., Magical Demonstrations. Education in Chemistry 2017, 54 (1), 17.

255 27. Smith, D. K., iTube, YouTube, WeTube: Social Media Videos in Chemistry Education and Outreach. J. Chem. Educ. 2014, 91 (10), 1594-1599.

28. Morrell, L.; Joyce, D., Interactive lectures: Clickers or personal devices? 2015; Vol. 4; DOI: 10.12688/f1000research.6207.1.

29. Van Dyke, A. R.; Smith-Carpenter, J. Bring Your Own Device: A Digital Notebook for Undergraduate Biochemistry Laboratory Using a Free, Cross-Platform Application. J. Chem. Educ. 2017, 94 (5), 656-661; DOI: $10.1021 /$ acs.jchemed.6b00622.

30. Herrington, J.; Herrington, A.; Mantei, J.; Olney, I.; Ferry, B. New Technologies, New Pedagogies: Mobile Learning in Higher Education; University of Wollongong: Wollongong, New South Wales, Australia, 2009; http://ro.uow.edu.au/newtech/ (accessed March 2018).

31. Almendro Vedia, V. G.; Natale, P.; Chen, S.; Monroy, F.; Rosilio, V.; López-Montero, I. iGUVs: Preparing Giant Unilamellar Vesicles with a Smartphone and Lipids Easily Extracted from Chicken Eggs. J. Chem. Educ. 2017, 94 (5), 644-649; DOI: 10.1021/acs.jchemed.6b00951.

32. Kehoe, E.; Penn, R. L., Introducing Colorimetric Analysis with Camera Phones and Digital Cameras: An Activity for High School or General Chemistry. J. Chem. Educ. 2013, 90 (9), 1191-1195.

33. Petruševski, V. M., Liquid Crystals Activity Revisited. J. Chem. Educ. 2007, 84 (9), 1429.

34. Warren, M.; Lewis, D. L., Liquid Crystals Activity. J. Chem. Educ. 2006, 83 (11), 1602.

35. Heo, I. [Display Dynamics] Why does AMOLED need polarizers? https://technology.ihs.com/509943/whyall-amoled-is-applying-polarizer-suppliers-of-amoled-polarizer-and-compensation-film-for-polarizertechnology-trend-of-amoled-use-polarizer (accessed March 2018).

36. Glasses for the current work were donated by the author after a screening of "Star Wars: Episode 7". A worldwide list of IMAX ${ }^{\circledR}$ screens can be accessed at https://en.wikipedia.org/wiki/List_of_IMAX_venues (accessed March 2018). 
1

2

3

4

37. Iskenderian-Epps, W. S.; Soltis, C.; O'Leary, D. J., Visual Isotope Effects: Demonstrating the Primary Kinetic Isotope Effect in the Chromium(VI) Oxidation of 2-Propanol-d8 and Methanol-d4. J. Chem. Educ. 2013, 90 (8), 1044-1047.

38. Campbell, D. J.; Rupe, S. B., Solvent Swelling and Optical Rotation Demonstrated on the Overhead Projector. J. Chem. Educ. 2000, 77 (7), 876.

39. Lin, S., Aromatic Bagels: An Edible Resonance Analogy. J. Chem. Educ. 2007, 84 (5), 779.

40. Even if the local magnetic field is affected by nearby instrumentation, or metal within the base of the visualiser, the relative angles will be unaffected if the phone is rotated in place.

41. Wong, K.-P., Optical rotary dispersion and circular dichroism. J. Chem. Educ. 1974, 51 (12), A573.

42. Schelz, J. P.; Purdy, W. C., An experiment in optical rotatory dispersion. J. Chem. Educ. 1964, 41 (12), 645.

43. Koubek, E.; Quinn, H., Change in optical rotation with wavelength. J. Chem. Educ. 1989, 66 (10), 853.

44. Compton, R. N.; Mahurin, S. M.; Zare, R. N., Demonstration of Optical Rotatory Dispersion of Sucrose. J. Chem. Educ. 1999, 76 (9), 1234.

45. Kinney, J. B.; Skinner, J. F. A., Device for easy demonstration of optical activity and optical rotatory dispersion. J. Chem. Educ. 1977, 54 (8), 494.

46. Hulburt, E. Natural and Magnetic Rotatory Dispersion of Optically Active Transparent Liquids. The Astrophysical Journal 1921, 54, 116. 